\title{
COMPARATIVE STUDY BETWEEN MEPERIDINE, KETAMINE, AND ONDASETRON FOR PROPHYLACTIC MANAGEMENT OF POST- SPINAL SHIVERING IN PATIENTS UNDERGOING LOWER ABDOMINAL SURGERIES
}

\author{
By
Tawfik M. Noor EL-Din*, Mohamed S. Sharaf ** Ahmed Mahmoud M. Algarhy *and Mahmoud S. Eisa*** \\ *Departments of Anesthesia and Intensive care, Faculty of Medicine, Al-Azhar University \\ (Cairo and Damietta) \\ ***Corresponding author details: E-mail:drmahmoud1890@yahoo.com
}

\begin{abstract}
Background: Shivering can be very unpleasant and physiologically stressful for the patient. It can be detrimental to patients with low cardio-respiratory reserve.

Objective: The study was done to compare the effect of Meperidine, Ketamine, and Ondansetron in prevention of post-spinal shivering.

Patients and Methods: This prospective randomized single blinded controlled clinical study was conducted on 120 patients under spinal anesthesia ASA I, II, undergoing lower abdominal and lower limb surgical procedures not exceeding 60 minutes. Patients were randomized into three equal groups. Group (A) received meperidine in a dose of $0.5 \mathrm{mg} / \mathrm{kg}$.i.v, Group(B) received ketamine in a dose of $0.25 \mathrm{mg} / \mathrm{kg}$.iv, and Group (C) received ondansetron in a dose of $4 \mathrm{mg}$. iv . All these drugs were given after subarachnoid block. Patients were evaluated by measuring shivering grade, HR, Systolic BP every 5 minutes for 60 minutes after interthecal injection. Shivering during spinal anesthesia was determindeded using a scale validated by Tsai and Chu. Blood pressure, heart rate and side effects were recorded.
\end{abstract}

Results: There were statistical significant differences between Meperidine, Ketamine, and Ondansetron as Meperidine has a superior effect in prevention of shivering. No changes in systolic BP and HR.

Conclusion: Meperidine and low dose ketamine $(0.25 \mathrm{mg} / \mathrm{kg})$ were significantly more effective than ondansetron for prevention of post-spinal shivering in patients undergoing lower abdominal surgeries.

Keywords: Meperidine, Ketamine, Ondansetron, sub arachnoid shivering.

\section{INTRODUCTION}

Shivering is one of the most important postoperative complications seen in clinical practice, (Morsali et al., 2017). It is an important concern in patients undergoing surgery under general anesthesia as well as regional anesthesia, i.e. spinal anesthesia or epidural anesthesia. The reported median incidence of shivering related to neuraxial anesthesia from different studies is $55 \%$ (Venkatraman et al., 2018). Because of its associated discomfort, distress, aggravation of pain, increased metabolic demands, and increased oxygen consumption. Prevention and treatment of postanesthesia shivering is an important 
component of perioperative management of the patient (Bagle et al., 2016).

To decrease the incidence and severity of post-spinal shivering many trials done either pharmacological or nonpharmacological. Non pharmacological like heavy blankets and reassurance. Pharmacological agents like clonidine, magnesium sulfate, dolasetron, ondansetron, ketamine and meperidine are used to control shivering (Kundra et al., 2017).

This study was designed to compare the effect of Meperidine, Ketamine, and Ondansetron in prevention of post-spinal shivering and determine which of these pharmacological interventions serves best to achieve therapeutic effect with minimal side effect.

\section{PATIENTS AND METHODS}

After approval by the local ethics committee, Faculty of Medicine, Al-Azhar University, and patients gave written consents, this prospective randomized single blinded controlled clinical study was conducted from February 2018 to May 2019 at Al-Azhar Hospital (New Dameitta) on 120 patients (21-60 years old) of both sexes ASA I, II, undergoing lower abdominal and lower limb surgeries not exceeding 60 minutes under spinal anesthesia. Exclusion criteria were ASA III, IV patients with body temperature above 38 or below 36.5 , history of convulsions, neuromuscular disorders, and patients with known allergies to the studied drugs. Patients were randomized into three equal groups, Group (A) received Meperidine in a dose of 0.5 $\mathrm{mg} / \mathrm{kg}$. IV, Group (B) received Ketamine in a dose of $0.25 \mathrm{mg} / \mathrm{kg}$. IV, and Group
(C) received Ondansetron in a dose of 4 mg. IV. All these drugs were given after subarachnoid block .

All studied patients were evaluated preoperatively by history, clinical examination, review of investigations, and premedicated with with oral diazepam (2mg) at night, and one hour before operation. Monitoring was done including electrocardiogram, SPO2, blood pressure, temperature, and baseline reading was recorded. Subarachnoid block was performed, using $25 \mathrm{G}$ disposable Quinke's spinal needle with $0.5 \%$ hyperbaric bupivacaine over $10 \mathrm{sec}$ in the inter-space L4-5 in sitting position.

Operating room temperature was maintained at $24^{\circ} \mathrm{C}-25^{\circ} \mathrm{C}$. Ringer Lactate solution preload $(10 \mathrm{ml} / \mathrm{kg} / \mathrm{h})$ and was maintained at $6 \mathrm{ml} / \mathrm{kg} / \mathrm{h}$ after spinal anesthesia.

Just after the intrathecal injection, one of the study drugs was given IV according to the group. These parameters were measured every 5 minutes for 60 minutes after intrathecal injection. Shivering incidence and grades, HR, and Systolic $\mathrm{BP}$, were recorded every 5 minutes. Shivering was graded using a scale similar to that validated by Tsai and Chu (2001).

Heart rate was maintained within 60 to $90 \mathrm{~b} / \mathrm{m}$. Hypotension was treated with intravenous Ringer's solution $(15 \mathrm{ml} / \mathrm{kg})$ and ephedrine (5-10 $\mathrm{mg}$ i.v) as needed. Bradycardia (HR $<60 / \mathrm{min}$.) was treated with atropine $(0.01-0.02 \mathrm{mg} / \mathrm{kg})$.

\section{Statistical analysis:}

Analysis of data was done by using SPSS (statistical program for social science version 16) as follows:Qualitative data were presented as 
numbers and percentages. Quantitative data were presented as mean \pm standard deviation. Qualitative data were analyzed by using Chi-square test. Quantitative data were analyzed by using one way ANOVA test HR\& BP were analyzed by repeatedmeasure ANOVA test. Significant data by one way ANOVA test were further analyzed by post-hoc analysis (Bonferroni correction) for detection of significant group. $\mathrm{P}<0.05$ was considered significant.

\section{RESULTS}

There was no significant difference in and type of surgery between groups (table the age, sex, weight, ASA physical status, $1)$.

Table(1): Demographic data and type of surgery in the studied groups

\begin{tabular}{|c|c|c|c|c|c|}
\hline \multicolumn{2}{|c|}{ Parameters } & $\begin{array}{c}\text { Group (A) } \\
\mathrm{N}=40\end{array}$ & $\begin{array}{c}\text { Group (B) } \\
\mathrm{N}=40\end{array}$ & $\begin{array}{c}\text { Group (C) } \\
\mathrm{N}=40\end{array}$ & p-value \\
\hline \multicolumn{2}{|c|}{ Age (years) } & $40.1 \pm 13.5$ & $43.33 \pm 11.12$ & $47.80 \pm 10.10$ & 0.014 \\
\hline \multicolumn{2}{|c|}{ Weight (Kg) } & $63.9 \pm 11.4$ & $60.93 \pm 7.31$ & $65.85 \pm 6.92$ & $>0.05$ \\
\hline \multicolumn{2}{|c|}{$\operatorname{Sex}(M / F)$} & $20 / 20$ & $25 / 15$ & $22 / 18$ & $>0.05$ \\
\hline \multicolumn{2}{|c|}{ ASA grade (1:2) } & $35 / 5$ & $38 / 2$ & $37 / 3$ & $>0.05$ \\
\hline \multirow{3}{*}{ 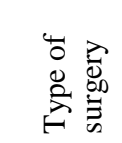 } & Vaginal hysterectomy & 15 patients & 10 patients & 10 patients & $>0.05$ \\
\hline & Appedictomy & 3 patints & 5 patients & 3 patients & $>0.05$ \\
\hline & Hernioplasty & 22 patients & 25patients & 27 patients & $>0.05$ \\
\hline
\end{tabular}

Shivering occurred in $3(7.5 \%)$ patients in group A, $4(10 \%)$ patients in group B, and $11(27.5 \%)$ patients in group C. So, there were significant differences between group A,B and group $\mathrm{C}$. But there was no significant difference between group $\mathrm{A}$ and group B (Table 2). 
TAWFIK M. NOOR EL-DIN et al.,

Table 2: Shivering grades in the studied groups

\begin{tabular}{|c|c|c|c|c|}
\hline$\underbrace{\text { Groups }}_{\text {Shivering grades }}$ & $\begin{array}{c}\text { Group A } \\
n=40\end{array}$ & $\begin{array}{c}\text { Group B } \\
n=40\end{array}$ & $\begin{array}{c}\text { Group C } \\
\mathrm{n}=\mathbf{4 0}\end{array}$ & P-value \\
\hline $\begin{array}{r}\text { After } 0 \text { min } \\
\text { Grade(0) } \\
\text { Grade(1) } \\
\text { Grade(2) } \\
\text { Grade(3) } \\
\text { Grade(4) }\end{array}$ & $\begin{array}{cc}35 & (87.5 \%) \\
5 & (12.5 \%) \\
0 & (0 \%) \\
0 & (0 \%) \\
0 & (0 \%)\end{array}$ & $\begin{array}{c}35(87.5 \%) \\
4(10 \%) \\
1(2.5 \%) \\
0 \quad(0 \%) \\
0 \quad(0 \%)\end{array}$ & $\begin{array}{l}30(75 \%) \\
3(7.5 \%) \\
7(17.5 \%) \\
0 \quad(0 \%) \\
0 \quad(0 \%)\end{array}$ & $<0.05$ \\
\hline $\begin{array}{l}\text { After } 5 \text { min } \\
\text { Grade }(0) \\
\text { Grade(1) } \\
\text { Grade(2) } \\
\text { Grade(3) } \\
\text { Grade(4) }\end{array}$ & $\begin{array}{l}28(70 \%) \\
10(25 \%) \\
1(2.5 \%) \\
1(2.5 \%) \\
0 \quad(0 \%)\end{array}$ & $\begin{array}{c}27(67.5 \%) \\
6(15 \%) \\
5(12.5 \%) \\
2(5 \%) \\
0 \quad(0 \%)\end{array}$ & $\begin{array}{l}28(70 \%) \\
5(12.5 \%) \\
3(7.5 \%) \\
4(10 \%) \\
0 \quad(0 \%)\end{array}$ & $<0.05$ \\
\hline $\begin{array}{l}\text { After } 10 \text { min } \\
\text { Grade }(0) \\
\text { Grade }(1) \\
\text { Grade( }(2) \\
\text { Grade(3) } \\
\text { Grade(4) }\end{array}$ & $\begin{array}{c}27(67.5 \%) \\
9(22.5 \%) \\
1(2.5 \%) \\
3(7.5 \%) \\
0 \quad(0 \%)\end{array}$ & $\begin{array}{c}27(67.5 \%) \\
7(17.5 \%) \\
3(7.5 \%) \\
2(5 \%) \\
1(2.5 \%)\end{array}$ & $\begin{array}{c}20(50 \%) \\
7(17.5 \%) \\
5(12.5 \%) \\
5(12.5 \%) \\
3(7.5 \%)\end{array}$ & $<0.05$ \\
\hline $\begin{array}{r}\text { After } 15 \text { min } \\
\text { Grade }(0) \\
\text { Grade }(1) \\
\text { Grade }(2) \\
\text { Grade }(3) \\
\text { Grade }(4)\end{array}$ & $\begin{array}{c}29(72.5 \%) \\
8(20 \%) \\
1(2.5 \%) \\
1(2.5 \%) \\
1(2.5 \%)\end{array}$ & $\begin{array}{c}27(67.5 \%) \\
6(15 \%) \\
3(7.5 \%) \\
2(5 \%) \\
2(5 \%) \\
\end{array}$ & $\begin{array}{c}18(45 \%) \\
7(17.5 \%) \\
4(10 \%) \\
6(15 \%) \\
5(12.5 \%)\end{array}$ & $<0.05$ \\
\hline $\begin{array}{c}\text { After } 20 \text { min } \\
\text { Grade(0) } \\
\text { Grade(1) } \\
\text { Grade(2) } \\
\text { Grade(3) } \\
\text { Grade(4) }\end{array}$ & $\begin{array}{c}29(72.5 \%) \\
8(20 \%) \\
0 \quad(0 \%) \\
1(2.5 \%) \\
2(5 \%)\end{array}$ & $\begin{array}{c}29(72.5 \%) \\
5(12.5 \%) \\
2(5 \%) \\
2(5 \%) \\
2(5 \%)\end{array}$ & $\begin{array}{c}15(37.5 \%) \\
9(22.5 \%) \\
5(12.5 \%) \\
5(12.5 \%) \\
6(15 \%)\end{array}$ & $<0.05$ \\
\hline $\begin{array}{r}\text { After } 25 \text { min } \\
\text { Grade }(0) \\
\text { Grade(1) } \\
\text { Grade(2) } \\
\text { Grade(3) } \\
\text { Grade(4) }\end{array}$ & $\begin{array}{c}29(72.5 \%) \\
8(20 \%) \\
1(2.5 \%) \\
1(2.5 \%) \\
1(2.5 \%)\end{array}$ & $\begin{array}{c}31(77.5 \%) \\
4(10 \%) \\
1(2.5 \%) \\
1(2.5 \%) \\
3(7.5 \%)\end{array}$ & $\begin{array}{c}15(37.5 \%) \\
7(17.5 \%) \\
7(17.5 \%) \\
4(10 \%) \\
7(17.5 \%)\end{array}$ & $<0.05$ \\
\hline $\begin{array}{r}\text { After } 30 \text { min } \\
\text { Grade(0) } \\
\text { Grade(1) } \\
\text { Grade(2) } \\
\text { Grade(3) } \\
\text { Grade(4) }\end{array}$ & $\begin{array}{c}31(77.5 \%) \\
6(15 \%) \\
1(2.5 \%) \\
1(2.5 \%) \\
1(2.5 \%)\end{array}$ & $\begin{array}{c}31(77.5 \%) \\
4(10 \%) \\
1(2.5 \%) \\
1(2.5 \%) \\
3(7.5 \%)\end{array}$ & $\begin{array}{c}15(37.5 \%) \\
7(17.5 \%) \\
7(17.5 \%) \\
4(10 \%) \\
7(17.5 \%)\end{array}$ & $<0.05$ \\
\hline $\begin{array}{r}\text { After } 45 \text { min } \\
\text { Grade }(0) \\
\text { Grade }(1) \\
\text { Grade(2) } \\
\text { Grade(3) } \\
\text { Grade(4) }\end{array}$ & $\begin{array}{c}33(82.5 \%) \\
3(17.5 \%) \\
1(2.5 \%) \\
1(2.5 \%) \\
2(5 \%)\end{array}$ & $\begin{array}{c}31(77.5 \%) \\
4(10 \%) \\
1(2.5 \%) \\
1(2.5 \%) \\
3(7.5 \%)\end{array}$ & $\begin{array}{c}15(37.5 \%) \\
7(17.5 \%) \\
7(17.5 \%) \\
4(10 \%) \\
7(17.5 \%)\end{array}$ & $<0.05$ \\
\hline $\begin{array}{r}\text { After } 60 \text { min } \\
\text { Grade }(0) \\
\text { Grade(1) } \\
\text { Grade(2) } \\
\text { Grade(3) } \\
\text { Grade(4) }\end{array}$ & $\begin{array}{c}33(82.5 \%) \\
3(17.5 \%) \\
1(2.5 \%) \\
1(2.5 \%) \\
2(5 \%)\end{array}$ & $\begin{array}{c}31(77.5 \%) \\
4(10 \%) \\
1(2.5 \%) \\
1(2.5 \%) \\
3(7.5 \%)\end{array}$ & $\begin{array}{c}15(37.5 \%) \\
7(17.5 \%) \\
7(17.5 \%) \\
1(2.5 \%) \\
10(25 \%)\end{array}$ & $<0.05$ \\
\hline
\end{tabular}

There was statistical significant difference between groups $\mathrm{A}, \mathrm{B}$ and group $\mathrm{C}$ regarding incidence of shivering (Table 3 ). 
COMPARATIVE STUDY BETWEEN MEPERIDINE, KETAMINE, ...

Table (3): Incidence of shivering among the studied groups

\begin{tabular}{|c|c|c|c|c|}
\hline Groups & Mepridine (A) & Ketamine (B) & Ondansetrone (C) & P-value \\
\hline $\begin{array}{c}\text { Shivering } \\
(\text { No \& } \%)\end{array}$ & $3(7.5 \%)$ & $4(10 \%)$ & $11(27.5 \%)$ & $<0.05^{*}$ \\
\hline
\end{tabular}

Heart rate showed no significant difference between the studied groups (Table 4).

Table (4): Heart rate ( beats per minute) in studied groups

\begin{tabular}{|c|c|c|c|c|}
\hline $\begin{array}{l}\text { Time } \\
\text { (minutes) }\end{array}$ & $\begin{array}{c}\text { Group (A) } \\
\text { Mean } \mathbf{\text { SD }} \\
\text { beat / min }\end{array}$ & $\begin{array}{c}\text { Group (B) } \\
\text { Mean } \mathbf{\text { SD }} \\
\text { beat / min }\end{array}$ & $\begin{array}{c}\text { Group ( ) } \\
\text { Mean } \mathbf{S D} \\
\text { beat } / \text { min }\end{array}$ & P \\
\hline (base line) & $83.2 \pm 5.7$ & $85.5 \pm 6.0$ & $83.5 \pm 6.2$ & $>0.5$ \\
\hline 5 & $88.2 \pm 8.6$ & $89.2 \pm 8.5$ & $87.2 \pm 8.3$ & $>0.5$ \\
\hline 10 & $78.1 \pm 8.8$ & $81.1 \pm 8.1$ & $79.1 \pm 8.8$ & $>0.5$ \\
\hline 15 & $79.0 \pm 8.6$ & $80.0 \pm 8.3$ & $78.0 \pm 8.2$ & $>0.5$ \\
\hline 20 & $75.0 \pm 10.1$ & $76.0 \pm 10$ & $73.0 \pm 9.8$ & $>0.5$ \\
\hline 25 & $72.0 \pm 9.0$ & $77.0 \pm 9.0$ & $71.0 \pm 8.0$ & $>0.5$ \\
\hline 30 & $75.8 \pm 8.8$ & $76.2 \pm 8.2$ & $77 \pm 8.2$ & $>0.5$ \\
\hline 35 & $73.9 \pm 7.8$ & $77.2 \pm 6.8$ & $78.1 \pm 5.4$ & $>0.5$ \\
\hline 40 & $72.2 \pm 6.8$ & $76.1 \pm 6.2$ & $74.2 \pm 5.8$ & $>0.5$ \\
\hline 45 & $72.4 \pm 6.5$ & $77.4 \pm 5.5$ & $76.4 \pm 4.5$ & $>0.5$ \\
\hline 50 & $71.8 \pm 6.3$ & $78.8 \pm 2.3$ & $73.8 \pm 5.3$ & $>0.5$ \\
\hline 55 & $70.9 \pm 6.3$ & $76.8 \pm 6.5$ & $75.8 \pm 6.2$ & $>0.5$ \\
\hline 60 & $72.8 \pm 6.4$ & $78.8 \pm 6.3$ & $76.8 \pm 6.5$ & $>0.5$ \\
\hline
\end{tabular}

In Group A, 4 patients were treated with atropine $0.01-0.02 \mathrm{mg} / \mathrm{kg}$ as $\mathrm{HR}$ decreased below 60/min. In Group C, 5 patients needed treatment by atropine, and 3 patients in Group B.
Systolic blood pressure showed no significant differences between groups (Table 5).

Table (5): Systolic BP ( $\mathrm{mm} \mathrm{Hg}$ ) in the studied groups

\begin{tabular}{|c|c|c|c|c|}
\hline Groups & $\begin{array}{c}\text { Group(A) } \\
\text { Mean } \pm \text { SD } \\
\mathbf{N = 4 0}\end{array}$ & $\begin{array}{c}\text { Group(B) } \\
\text { Mean } \pm \text { SD } \\
\mathbf{N = 4 0}\end{array}$ & $\begin{array}{c}\text { Group(C) } \\
\text { Mean } \pm \text { SD } \\
\mathbf{N = 4 0}\end{array}$ & p \\
\hline (base line) & $122.3 \pm 13.3$ & $123.3 \pm 13.3$ & $120.3 \pm 13.3$ & $>0.5$ \\
\hline 5 min & $120.7 \pm 10.9$ & $120.7 \pm 10.9$ & $121.7 \pm 10.9$ & $>0.5$ \\
\hline $10 \mathrm{~min}$ & $113.3 \pm 10.7$ & $114.3 \pm 10.7$ & $113.3 \pm 10.7$ & $>0.5$ \\
\hline $15 \mathrm{~min}$ & $110.4 \pm 5.6$ & $118.4 \pm 5.6$ & $110.4 \pm 5.6$ & $>0.5$ \\
\hline $20 \mathrm{~min}$ & $111.7 \pm 10.1$ & $117.7 \pm 10.1$ & $111.7 \pm 10.1$ & $>0.5$ \\
\hline $25 \mathrm{~min}$ & $117.9 \pm 8.7$ & $117.9 \pm 8.7$ & $117.9 \pm 8.7$ & $>0.5$ \\
\hline $30 \mathrm{~min}$ & $118.8 \pm 9.4$ & $120.8 \pm 9.4$ & $118.8 \pm 9.4$ & $>0.5$ \\
\hline $35 \mathrm{~min}$ & $119.5 \pm 8.2$ & $119.5 \pm 8.2$ & $116.5 \pm 8.2$ & $>0.5$ \\
\hline $40 \mathrm{~min}$ & $118.2 \pm 6.2$ & $119.2 \pm 6.2$ & $116.2 \pm 6.2$ & $>0.5$ \\
\hline $45 \mathrm{~min}$ & $119.3 \pm 5.6$ & $119.3 \pm 5.6$ & $115.3 \pm 5.6$ & $>0.5$ \\
\hline $50 \mathrm{~min}$ & $118.8 \pm 4.2$ & $119.8 \pm 4.2$ & $118.8 \pm 4.2$ & $>0.5$ \\
\hline $55 \mathrm{~min}$ & $119.2 \pm 6.7$ & $119.2 \pm 6.7$ & $119.2 \pm 6.7$ & $>0.5$ \\
\hline $60 \mathrm{~min}$ & $121.4 \pm 5.6$ & $121.4 \pm 5.6$ & $120.4 \pm 5.6$ & $>0.5$ \\
\hline
\end{tabular}


Diastolic blood pressure showed no significant differences between groups (Table $6)$.

Table (6): Diastolic BP ( $\mathrm{mm} \mathrm{Hg}$ ) in the studied groups .

\begin{tabular}{|c|c|c|c|c|}
\hline Groups & $\begin{array}{c}\text { Group(A) } \\
\text { Mean } \mathbf{\pm S D} \\
\mathbf{N = 4 0}\end{array}$ & $\begin{array}{c}\text { Group(B) } \\
\text { Mean } \mathbf{4 S D} \\
\mathbf{N = 4 0}\end{array}$ & $\begin{array}{c}\text { Group(C) } \\
\text { Mean } \pm \text { SD } \\
\mathbf{N = 4 0}\end{array}$ & P \\
\hline (base line) & $74.1 \pm 7.7$ & $75.1 \pm 7.6$ & $75.1 \pm 7.7$ & $>0.5$ \\
\hline $5 \mathrm{~min}$ & $73.9 \pm 6.4$ & $74.9 \pm 5.4$ & $73.9 \pm 6.4$ & $>0.5$ \\
\hline $10 \mathrm{~min}$ & $70.1 \pm 4.9$ & $73.1 \pm 3.8$ & $71.1 \pm 4.9$ & $>0.5$ \\
\hline $15 \mathrm{~min}$ & $69.6 \pm 5.3$ & $71.6 \pm 3.3$ & $69.6 \pm 5.3$ & $>0.5$ \\
\hline $20 \mathrm{~min}$ & $68.6 \pm 5.3$ & $71.6 \pm 2.3$ & $69.6 \pm 5.3$ & $>0.5$ \\
\hline $25 \mathrm{~min}$ & $68.6 \pm 6.3$ & $73.6 \pm 4.3$ & $69.6 \pm 6.3$ & $>0.5$ \\
\hline $30 \mathrm{~min}$ & $69.6 \pm 5.5$ & $71.8 \pm 2.5$ & $69.6 \pm 5.5$ & $>0.5$ \\
\hline $35 \mathrm{~min}$ & $67.7 \pm 6.4$ & $73.7 \pm 5.4$ & $68.7 \pm 6.4$ & $>0.5$ \\
\hline $40 \mathrm{~min}$ & $70.3 \pm 4.7$ & $74.3 \pm 5.7$ & $70.3 \pm 4.7$ & $>0.5$ \\
\hline $45 \mathrm{~min}$ & $64.2 \pm 6.2$ & $75.2 \pm 5.8$ & $66.2 \pm 6.2$ & $>0.5$ \\
\hline $50 \mathrm{~min}$ & $76.9 \pm 3.8$ & $77.2 \pm 5.8$ & $76.9 \pm 3.8$ & $>0.5$ \\
\hline $55 \mathrm{~min}$ & $72.7 \pm 4.8$ & $70.6 \pm 4.8$ & $72.7 \pm 4.8$ & $>0.5$ \\
\hline $60 \mathrm{~min}$ & $70.6 \pm 4.6$ & $73.6 \pm 5.2$ & $70.6 \pm 4.6$ & $>0.5$ \\
\hline
\end{tabular}

Hypotension was seen in 5 cases $(12.5 \%)$ in Group A, 3 cases $(7.5 \%)$ in group B and 6 cases $(15 \%)$ in group $\mathrm{C}$.

There was no significant difference between groups regarding hypotension, nausea, vomiting, and hallucinations, but there was a significant difference between $\mathrm{C}$ group and $\mathrm{A}, \mathrm{B}$ groups regarding sedation (Table 7).

Table(7): Side effects among studied groups

\begin{tabular}{|l|c|c|c|c|}
\hline & Group (A) (n=40) & $\begin{array}{c}\text { Group (B) } \\
(\mathbf{n = 4 0 )}\end{array}$ & $\begin{array}{c}\text { Group (C) } \\
(\mathbf{n = 4 0 )}\end{array}$ & P value \\
\hline Side effects & 5 & 3 & 6 & $>0.05$ \\
\hline Nausea & 4 & 3 & 1 & $>0.05$ \\
\hline Vomiting & 3 & 2 & 1 & $>0.05$ \\
\hline Sedation & 37 & 38 & 0 & $<0.001$ \\
\hline Hallucinations & 0 & 2 & 0 & $>0.05$ \\
\hline
\end{tabular}

\section{DISCUSSION}

Shivering is one of the most important postoperative complications seen in clinical practice (Morsali et al., 2017). It is an important concern in patients undergoing surgery under general anesthesia as well as regional anesthesia, i.e. spinal anesthesia or epidural anesthesia. The reported median incidence of shivering related to neuraxial anesthesia from different studies is $55 \%$ (Venkatraman et al., 2018). Because of its associated discomfort, distress, aggravation of pain, increased metabolic demands, and increased oxygen consumption. Prevention and treatment of postanesthesia shivering is an important component of perioperative management of the patient (Bagle et al., 2016).

Shivering considered significant when the patient shivered at least to grade 3 after 15 minutes of spinal anesthesia (Tsai and Chu, 2001). From this concept, we tried in this study to clarify the antishivering effect of Mepridine, Ketamine and Ondanstron. In our study, regarding significant shivering, it occurred 
in $7.5 \%$ of patients of group A, $10 \%$ of patients of group B, and $27.5 \%$ of patients of group C. So, there was significant difference between group A, B and group $\mathrm{C}$. There was no significant difference between group A and group B. This agreed with Shakya et al. (2010) who showed that low-dose ketamine and ondansetron are effective in the prevention of shivering compared with placebo. In this study, ketamine was significantly more effective than ondansetron in controlling shivering after spinal anesthesia that, can be partly confirming the results of our study. Our study also agreed with The study done by Browning et al. (2013) who found no significant difference in the incidence of post spinal shivering between ondansetron or saline administered before establishing combined spinal/epidural anesthesia in parturient underwent cesarean section. Our study agreed with Badawy and Mokhtar (2017) who studied the role of ondansetron in prevention of post-spinal shivering (PSS) in obstetric patients. They found that there was a statistically significant higher incidence of shivering in group (B), compared to group (C). So, there was nearly the same percent of shivering in group (C).

Our study did not agree with Safavi and Colleagues (2014) who stated that midazolam is effective in preventing shivering after spinal anesthesia. This disagreement may be due to their larger dose of ondansetron

As regard HR, in group (A), 4 patients treated with atropine $(0.01-0.02 \mathrm{mg} / \mathrm{kg})$ as HR decreased below 60/min. In group (C), 5 patients needed treatment, and 3 patients in group $(\mathrm{C})$.
As regard side effects, hypotension was seen in $12.5 \%$ in group (A), $7.5 \%$ in group (B) and $15 \%$ in group (C). Mild sedation was seen in $95 \%$ in group (B), $92.5 \%$ in group (A), and none in group (C) most of them were sedated to grade $1 \& 2$ and not reach grade 3 . It was obvious that there was no sedation seen in ondansetron. Shakya et al., (2010) stated that hallucinations were not noted in Ketamine group.

Nausea was seen in 4 patients in group (A), 3 in group (B) and 1 patient in group (C). Vomiting was seen in 3 patients in group (A), 2 in group (B) and 1 patient in group (C). Such patients were administered i.v. metoclopramide $10 \mathrm{mg}$.

\section{CONCLUSION}

Meperidine and low dose of ketamine were more effective than ondansetron for prevention of post-spinal shivering with minimal hemodynamic effects, but more sedation in patients undergoing lower abdominal surgeries.

\section{REFERENCES}

1. Bagle A, Thatte W, Khatavkar S, Choudhary S and Vagasia B. (2016): Oral clonidine for shivering prophylaxis in patients undergoing elective urological surgeries under subarachnoid anesthesia. Int J Health Allied Sci., 5:143-7.

2. Browning RM, Fellingham WH, O'Loughlin EJ and Brown NA (2013): Prophylactic ondansetron does not prevent shivering or decrease shivering severity during cesarean delivery under combined spinal epidural anesthesia: a randomized trial. RegAnesth Pain Med., 38:39-43.

3. Badawy and Mokhtar (2017): The role of ondansetron in prevention of post-spinal shivering (PSS) in obstetric patients. Egyptian Journal of Anaesthesia, 33,L1,: 933. 
4. Kundra TS, Kuthiala G, Shrivastava A, Kaur P. (2017): A comparative study on the efficacy of dexmedetomidine and tramadol on post-spinal anesthesia shivering. Saudi J Anaesth., 11:2-8.

5. Morsali SF, Movasseghi G, Kiaee MM, Ghorbanloo M, Mohaghegh MR, and Morsali A, (2017): Clonidine versus tramadol for postanesthetic shivering: A randomized clinical trial study. Biomed Res Ther. 4:1716-32.

6. Shakya S, Chaturvedi A and Sah BP. (2010): Prophylactic low dose ketamine and ondansetron for prevention of shivering during spinal anaesthesia. J AnaesthesiolClinPharmacol.;26(4):465-9.

7. Safavi M, Honarmand A, Negahban M and Attari M. (2014): Prophylactic effects of intrathecalMeperidine and intravenous Ondansetron on shivering in patients undergoing lower extremity orthopedic surgery under spinal anesthesia. Journal of research in pharmacy practice. 3(3): 94.

8. Tsai YC and Chu KS. (2001): A comparison of tramadol, amitriptyline, andmeperidine for post epidural anesthetic shivering. Anesthesia\& Analgesia 93(5):1288-92.

9. Venkatraman R, Karthik K, Pushparani A, and Mahalakshmi A. (2018): A prospective, randomized, double-blinded control study on comparison of tramadol, clonidine and dexmedetomidine for post spinal anesthesia shivering. Rev Bras Anestesiol, 68:42-8. 
مقارنة بين الميبريدين و الكيتامين و اللأوندانسيترون للوقاية

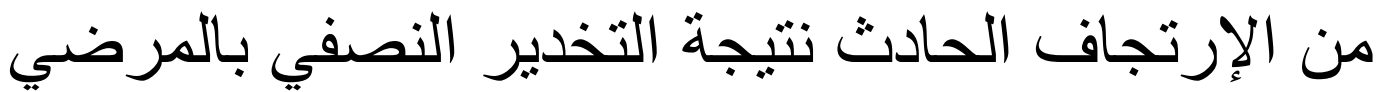

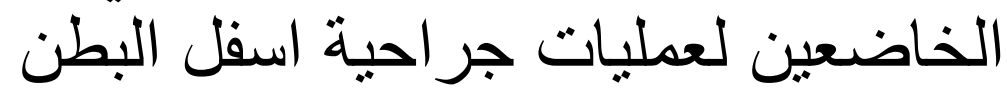

توفيق محمد نور الدين، محمد سامي شرف، أحمد محمود الجارحي، محمود سعيد عباس عيسى

$$
\text { أقسام التخدير والعناية المركزة ـ كلية الطب (القاهرة ـ دمياط) }
$$

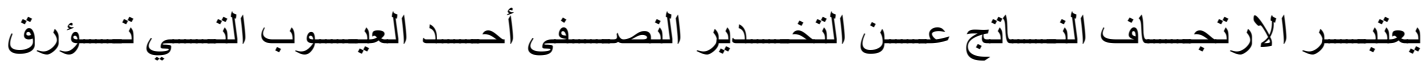

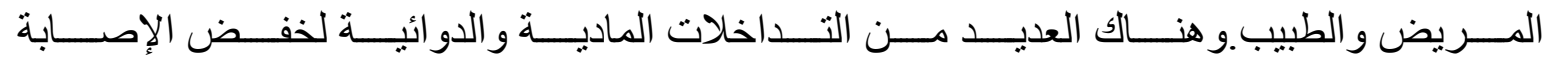
و الحد من الارتجاف الناتج عن التخدير النصفي.

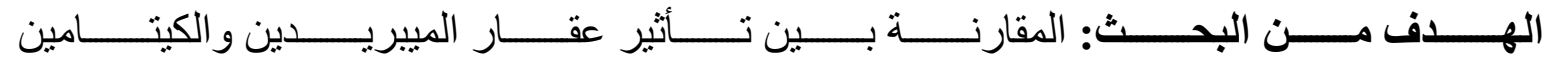
والأوندانسيترون لمنع الارتجاف الناتج عن التخدير النصفي.

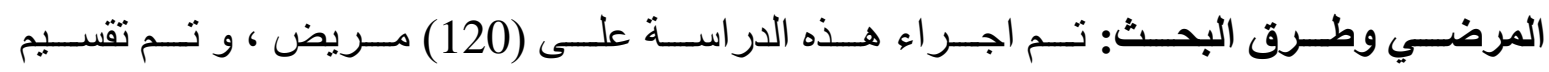
المرضى بشكل عشو ائي إلى ثلاث مجمو عات منساوية:

• المجمو عة الأولى : خضعت للحقن من المييريدين (0.5مجم/كجم). • المجمو عة الثانية : خضعت للحقن من الكيتامين (0.25 مجم/كجم). • المجمو عة الثالثة : خضعت للحقن من الأوندانسيترون (4 مجم).

وتـــم اســـتبعاد المرضــــى الـــذين يعـــانون مـــن الأمـــر اض العصــــبية و العضـــلية

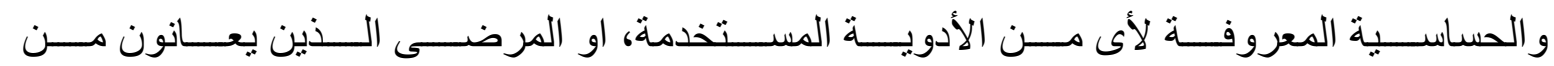

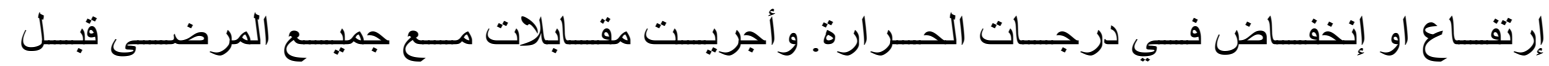

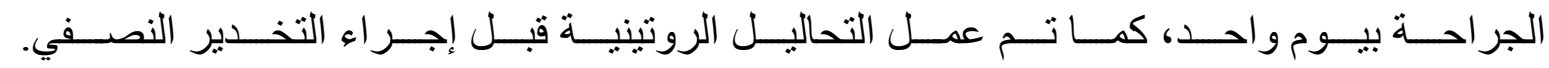

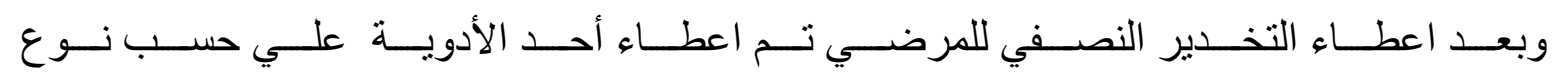

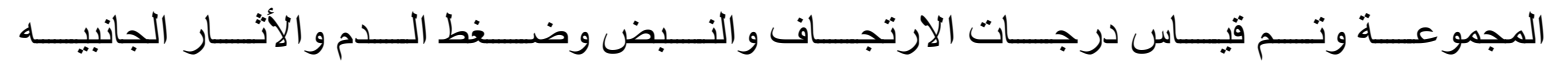
للأدوية مثل إنخفاض ضغط الدم و الغتيان و القئ و الهلوسة. 


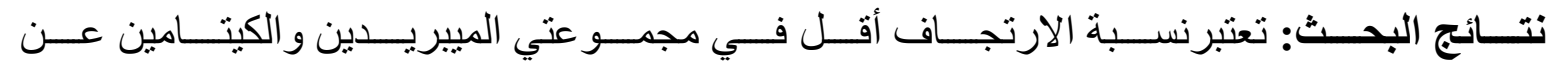

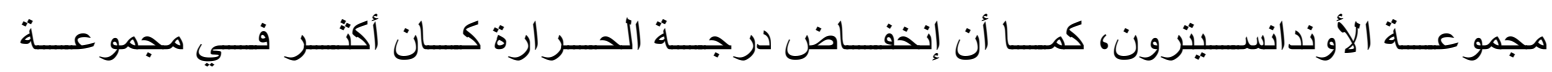

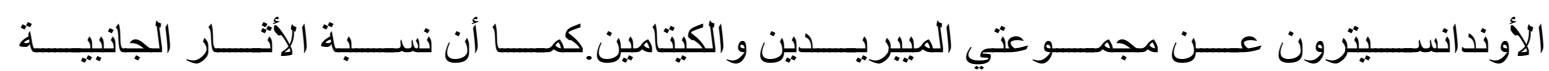
أقل في مجمو عة الأوندانسيترون.

الاســتنتاج: يعتبــر حقـن كـلاً مــن عقـاري الميبريــدين والكيتــامين أكثــر فعاليــة مــن عقــار الأوندانسيترون للحد من الارتجاف الناتج عن التخدير النصفي. 